\title{
Integral-Based Identification of Patient Specific Parameters for a Minimal Cardiac Model
}

\author{
C. E. Hann ${ }^{1}$, J. G. Chase ${ }^{2}$, G. M. Shaw ${ }^{3}$ \\ Department of Mechanical Engineering \\ University of Canterbury \\ Private Bag 4800 \\ Christchurch \\ New Zealand
}

Email: Chris.Hann@canterbury.ac.nz

\footnotetext{
${ }^{1}$ Research Associate, New Zealand Science and Technology Postdoctoral Fellow, Dept. of Mech. Eng

${ }^{2}$ Assoc. Prof./Reader, Dept of Mech. Eng

${ }^{3}$ Consultant, Christchurch Hospital Dept of Intensive Care Medicine,

Christchurch, New Zealand
} 


\begin{abstract}
A minimal cardiac model has been developed which accurately captures the essential dynamics of the cardiovascular system (CVS). However, identifying patient specific parameters with the limited measurements often available, hinders the clinical application of the model for diagnosis and therapy selection. This paper presents an integral based parameter identification method for fast, accurate identification of patient specific parameters using limited measured data. The integral method turns a previously non-linear and non-convex optimization problem into a linear and convex identification problem.

The model includes ventricular interaction and physiological valve dynamics. A healthy human state and two disease states, Valvular Stenosis and Pulmonary Embolism, are used to test the method. Parameters for the healthy and disease states are accurately identified using only discretized flows into and out of the two cardiac chambers, the minimum and maximum volumes of the left and right ventricles, and the pressure waveforms through the aorta and pulmonary artery. These input values can be readily obtained non-invasively using echo-cardiography and ultra-sound, or invasively via catheters that are often used in Intensive Care.

The method enables rapid identification of model parameters to match a particular patient condition in clinical real time (3-5 minutes) to within a mean value of $4-8 \%$ in the presence of $5-15 \%$ uniformly distributed measurement noise. The specific changes made to simulate each disease state are correctly identified in each case to within $5 \%$ without false identification
\end{abstract}


of any other patient specific parameters. Clinically, the resulting patient specific model can then be used to assist medical staff in understanding, diagnosis and treatment selection.

\section{Introduction}

Optimizing haemodynamics in the critically ill is an important task for intensive care staff who must filter and integrate a diverse range of information about a patient's circulation to provide the best care. Often this information is time-varying, incomplete and/or confusing leaving medical professionals to rely on their experience and intuition to identify and treat a patient's condition. A patient specific model enables therapeutic choices to be tested and, with patient specific parameter identification, can aid diagnosis of subtle haemodynamic behaviors, enabling better, more consistent care.

A minimal model developed by Smith et al $[1 ; 2 ; 3]$, has been shown to provide magnitudes and trends in agreement with existing data for a variety of physiologically verified test cases and disease states in CVS function [2;3]. This model uses a minimal number of parameters and equations where other models in the literature are more complex $[1 ; 3]$. To use this model to assist medical staff in diagnosis and treatment, a fast, accurate method for identifying patient specific parameters is required.

Ideally, the means of identifying the parameters should be convex to avoid finding false solutions. However, the dynamics of this model do not lend themselves to a convex problem [3]. The most commonly used method for identifying parameters in a physiological model described by differential equa- 
tions is non-linear regression [4]. This method, as with any gradient based optimization, involves solving the differential equations each time the parameters are updated. The problem with this approach is that even if the differential equation is linear in the parameters the numerical (or analytical) solution will, in general, be non-linear in the parameters. Thus, the optimization is non-linear and there is no guarantee of finding the correct solution. In addition, the numerical solution procedure can be very computationally intense, especially if long periods of data are being fit $[5 ; 6]$. The cardiac model in this research needs to be run each time for many heart beats to ensure a steady state solution is obtained so that accurate gradients can be found [3]. This latter requirement severely limits the number of optimization iterations available to find a solution in a clinically useful time period.

In this paper, the parameter identification optimization is formulated in terms of integrals, which enables a set of linear equations in the parameters to be created. This approach leads to a unique linear least squares solution so the optimization is convex and computationally fast. In addition, the differential equations are not required to be solved, which significantly further reduces the computation required. As a result, the computations required can be readily performed on a standard PC in 0-3 minutes, a clinically useful time period.

Equally importantly, for this research, the measured output data satisfies the criteria that it is either readily or reasonably available in the Intensive Care Unit. Hence, the methods developed can be directly applied clinically without excessive computational or measurement requirements. 


\section{Model and Methodology}

\subsection{Single elastic chamber}

The differential equations for the single elastic chamber shown in Figure 1 with inertia and upstream and downstream pressures $P_{1}$ and $P_{3}$, are defined $[1]:$

$$
\begin{aligned}
\dot{V} & =Q_{1}-Q_{2} \\
\dot{Q}_{1} & =\frac{P_{1}-P_{2}-Q_{1} R_{1}}{L_{1}} \\
\dot{Q}_{2} & =\frac{P_{2}-P_{3}-Q_{2} R_{2}}{L_{2}}
\end{aligned}
$$

where $Q_{1}$ and $Q_{2}$ are the flows in and out, $L_{1}$ and $L_{2}$ are inertances of the blood, $R_{1}$ and $R_{2}$ are resistances. The driving pressure in the chamber is defined:

$$
\begin{aligned}
P_{2} & =e(t) E_{\mathrm{es}}\left(V-V_{d}\right)+(1-e(t)) P_{0}\left(e^{\lambda\left(V-V_{0}\right)}-1\right), \\
e(t) & =e^{-80(t-0.375)^{2}}
\end{aligned}
$$

where $E_{\text {es }}$ is elastance, $V_{d}$ is volume at zero pressure, $e(t)$ is a driving function that simulates ventricular contraction and $P_{0}, \lambda$, and $V_{0}$ define gradient, curvature and volume at zero pressure of the EDPVR curve in the cardiac cycle shown in Figure 2 [1].

Equations (1) and (2) are solved when $Q_{1}>0$, during the filling stage, and Equations (1) and (3) are solved when $Q_{2}>0$, during the ejection stage. This model has an open on pressure, close on flow valve law as shown in Figure $2[2 ; 3]$. Figure 2 shows the states used for each portion of the cardiac cycle. 


\subsection{Integral method and Full model}

For the full CVS model shown in Figure 3 flows into and out of the left and right ventricles are as described for the single chamber in Equations (2)-(4), and the systemic and pulmonary flows are defined using Poiseuille's equation $[3 ; 7 ; 8]$,

$$
\begin{aligned}
Q_{\mathrm{sys}} & =\frac{P_{\mathrm{ao}}-P_{\mathrm{vc}}}{R_{\mathrm{sys}}} \\
Q_{\mathrm{pul}} & =\frac{P_{\mathrm{pa}}-P_{\mathrm{pu}}}{R_{\mathrm{pul}}}
\end{aligned}
$$

For this research, it is assumed that

- the flow profiles in the cardiac chambers

- the minimum and maximum volumes of the left and right ventricles

- the pressure waveforms through the aorta and pulmonary artery

can be measured using echo-cardiography or ultra-sound, (e.g. [9]-[17]). These assumptions represent a use of limited data to identify the full model. The volume in the left and right ventricles are described, using Figure 3, by the differential equations:

$$
\begin{aligned}
& \dot{V}_{\mathrm{lv}}=Q_{\mathrm{mt}}-Q_{\mathrm{av}} \\
& \dot{V}_{\mathrm{rv}}=Q_{\mathrm{tc}}-Q_{\mathrm{pv}}
\end{aligned}
$$

For the left ventricle, integrating Equation (8) from $t_{e}$ to $t$ during ejection and from $t_{f}$ to $t$ during filling gives:

$$
\begin{aligned}
V_{\mathrm{lv}}(t) & =V_{\mathrm{lv}}\left(t_{e}\right)-\int_{t_{e}}^{t} Q_{\mathrm{av}} d t, & & t_{e} \leq t \leq t_{f} \\
& =V_{\mathrm{lv}}\left(t_{f}\right)+\int_{t_{f}}^{t} Q_{\mathrm{mt}} d t, & & t_{f} \leq t \leq t_{e}+\tau
\end{aligned}
$$


where, as illustrated in Figure 2:

$$
\begin{aligned}
& t_{e} \equiv \text { beginning of ejection stage } \\
& t_{f} \equiv \text { beginning of filling stage } \\
& \tau \equiv \text { period of heart beat }
\end{aligned}
$$

Thus,

- $V_{\mathrm{lv}}\left(t_{e}\right)=V_{\mathrm{lv}, \max } \equiv$ maximum volume of the left ventricle

- $V_{\mathrm{lv}}\left(t_{f}\right)=V_{\mathrm{lv}, \min } \equiv$ minimum volume of the left ventricle

The right ventricle can be treated similarly.

The differential equations describing the flows through the aortic valve and mitral valve on either side of the left ventricle can be written for the full model [1]-[3]:

$$
\begin{aligned}
L_{\mathrm{av}} \dot{Q}_{\mathrm{av}} & =P_{\mathrm{lv}}-P_{\mathrm{ao}}-R_{\mathrm{av}} Q_{\mathrm{av}} \\
L_{\mathrm{mt}} \dot{Q}_{\mathrm{mt}} & =P_{\mathrm{pu}}-P_{\mathrm{lv}}-R_{\mathrm{mt}} Q_{\mathrm{mt}} \\
P_{\mathrm{lv}} & =P_{\mathrm{lvf}}+P_{\mathrm{peri}} \\
P_{\mathrm{lvf}} & =e(t) E_{\mathrm{es}, \mathrm{lvf}} V_{\mathrm{lvf}}+(1-e(t)) P_{0, \mathrm{lvf}}\left(e^{\lambda_{\mathrm{lvf}} V_{\mathrm{lvf}}}-1\right) \\
V_{\mathrm{lvf}} & =V_{\mathrm{lv}}-V_{\mathrm{spt}} \\
P_{\mathrm{peri}} & =P_{\mathrm{pcd}}+P_{\mathrm{th}} \\
P_{\mathrm{pcd}} & =P_{0, \mathrm{pcd}}\left(e^{\lambda_{\mathrm{pcd}}\left(V_{\mathrm{pcd}}-V_{0, \mathrm{pcd}}\right)}-1\right)
\end{aligned}
$$




$$
\begin{aligned}
& V_{\mathrm{pcd}}=V_{\mathrm{lv}}+V_{\mathrm{rv}} \\
& P_{\mathrm{ao}}=E_{\mathrm{ao}} V_{\mathrm{ao}} \\
& P_{\mathrm{pu}}=E_{\mathrm{pu}} V_{\mathrm{pu}}+P_{\mathrm{th}} \\
& \dot{V}_{\mathrm{ao}}=Q_{\mathrm{av}}-Q_{\mathrm{sys}} \\
& \dot{V}_{\mathrm{pu}}=Q_{\mathrm{pul}}-Q_{\mathrm{mt}}
\end{aligned}
$$

where $E_{j}$ denotes the $j^{\text {th }}$ chamber elastance in Figure $3, Q_{\text {sys }}$ and $Q_{\text {pul }}$ are given by Equations (6) and (7), and the other variables are defined in Figure 3. The pressures in the pulmonary artery $\left(P_{\mathrm{pu}}\right)$ and vena cava $\left(P_{\mathrm{vc}}\right)$ chambers in Figure 3 are defined:

$$
\begin{aligned}
& P_{\mathrm{vc}}=E_{\mathrm{vc}} V_{\mathrm{vc}} \\
& P_{\mathrm{pa}}=E_{\mathrm{pa}} V_{\mathrm{pa}}+P_{\mathrm{th}}
\end{aligned}
$$

\subsection{Integral equations for the full model}

Equations (14)-(25) can now be reformulated in terms of integrals. For fixed values of generic parameters $P_{0, \mathrm{pcd}}, \lambda_{\mathrm{pcd}}, P_{\mathrm{th}}$ and $V_{0, \mathrm{pcd}}, P_{\text {peri }}$ is completely determined by the volume profiles $V_{\mathrm{lv}}(t)$ given by Equation (10) and the similar equation for $V_{\mathrm{rv}}(t)$. The rest of this section develops the integral equations for the system identification problem for the left ventricle, and the aortic (ao) and pulmonary (pu) chambers in Figure 3. The exactly same approach can be applied to the upper half of the model. 
Integrating Equations (24) and (25) from 0 to $t$ gives:

$$
\begin{aligned}
V_{\mathrm{ao}} & =V_{\mathrm{ao}}(0)+\int_{0}^{t}\left(Q_{\mathrm{av}}-Q_{\mathrm{sys}}\right) d t \\
V_{\mathrm{pu}} & =V_{\mathrm{pu}}(0)+\int_{0}^{t}\left(Q_{\mathrm{pul}}-Q_{\mathrm{mt}}\right) d t \\
\Longrightarrow \quad P_{\mathrm{ao}} & =P_{\mathrm{ao} 0}+E_{\mathrm{ao}} \int_{0}^{t}\left(Q_{\mathrm{av}}-Q_{\mathrm{sys}}\right) d t \\
P_{\mathrm{pu}} & =P_{\mathrm{pu} 0}+E_{\mathrm{pu}} \int_{0}^{t}\left(Q_{\mathrm{pul}}-Q_{\mathrm{mt}}\right) d t+P_{\mathrm{th}}
\end{aligned}
$$

where the only unknowns are $E_{\mathrm{ao}}, E_{\mathrm{pu}}, P_{\mathrm{pu}}, V_{\mathrm{ao}}, V_{\mathrm{pu}}, V_{\mathrm{ao}}(0), V_{\mathrm{pu}}(0), P_{\mathrm{ao} 0}$ and $P_{\mathrm{pu} 0}$, assuming $Q_{\mathrm{av}}, Q_{\mathrm{mt}}$ and $P_{\mathrm{ao}}$ are measured, $P_{\mathrm{th}}$ is given and $Q_{\mathrm{sys}}$ and $Q_{\text {pul }}$ are determined independently to this formulation as described later. The terms $P_{\text {ao0 }}$ and $P_{\text {ao0 }}$ are defined:

$$
\begin{gathered}
P_{\mathrm{ao} 0}=E_{\mathrm{ao}} V_{\mathrm{ao}}(0) \\
P_{\mathrm{pu} 0}=E_{\mathrm{pu}} V_{\mathrm{pu}}(0) .
\end{gathered}
$$

In practice, given $E_{\mathrm{ao}}$ and $E_{\mathrm{pu}}, V_{\mathrm{pu}}\left(t_{0}\right)$ and $V_{\mathrm{ao}}\left(t_{0}\right)$ can be determined only approximately using Equations (32) and (33), due to noise in the measurements. Therefore, $P_{\mathrm{ao} 0}$ and $P_{\mathrm{pu} 0}$ are treated as extra unknown parameters. The flows $Q_{\mathrm{sys}}$ and $Q_{\mathrm{pul}}$ and the parameters $E_{\mathrm{ao}}, E_{\mathrm{pa}}, E_{\mathrm{pu}}, E_{\mathrm{vc}}, R_{\mathrm{sys}}$ and $R_{\mathrm{pul}}$ can then be determined from the measured wave forms of $P_{\mathrm{ao}}$ and $P_{\mathrm{pa}}$, and the flows in the left and right ventricle chambers.

Substituting Equations (16), (17), (30) and (31) into Equations (14) and (15) gives equations with the measured $Q_{\mathrm{av}}$ and $Q_{\mathrm{mt}}$ values on the left hand 
right hand sides.

$$
\begin{aligned}
L_{\mathrm{av}} \dot{Q}_{\mathrm{av}}= & E_{\mathrm{es}, \mathrm{lvf}} e(t) V_{\mathrm{lvf}}+P_{0, \mathrm{lvf}}(1-e(t))\left(e^{\lambda_{\mathrm{lvf}} V_{\mathrm{lvf}}}-1\right)+P_{\mathrm{peri}} \\
& -P_{\mathrm{ao} 0}-E_{\mathrm{ao}} \int_{0}^{t}\left(Q_{\mathrm{av}}-Q_{\mathrm{sys}}\right) d t-R_{\mathrm{av}} Q_{\mathrm{av}} \\
L_{\mathrm{mt}} \dot{Q}_{\mathrm{mt}}= & P_{\mathrm{pu} 0}+E_{\mathrm{pu}} \int_{0}^{t}\left(Q_{\mathrm{pul}}-Q_{\mathrm{mt}}\right) d t+P_{\mathrm{th}}-P_{\mathrm{peri}} \\
& -E_{\mathrm{es}, \mathrm{lvf}} e(t) V_{\mathrm{lvf}}-P_{0, \mathrm{lvf}}(1-e(t))\left(e^{\lambda_{\mathrm{lvf}} V_{\mathrm{lvf}}}-1\right)
\end{aligned}
$$

Integrating Equation (34) from $t_{e}$ to $T$ yields equations with measured values and unknowns on the left and right hand sides.

$$
\begin{aligned}
L_{\mathrm{av}}\left(Q_{\mathrm{av}}(T)-Q_{\mathrm{av}}\left(t_{e}\right)\right) & =E_{\mathrm{es}, \mathrm{lvf}} \int_{t_{e}}^{T} e V_{\mathrm{lvf}} d T+P_{0, \mathrm{lvf}} \int_{t_{e}}^{T}(1-e)\left(e^{\lambda_{\mathrm{lvf}} V_{\mathrm{lvf}}}-1\right) d T \\
& +\int_{t_{e}}^{T} P_{\mathrm{peri}} d T-P_{\mathrm{ao} 0}\left(T-t_{e}\right) \\
& -E_{\mathrm{ao}} \int_{t_{e}}^{T}\left(\int_{0}^{t}\left(Q_{\mathrm{av}}-Q_{\mathrm{sys}}\right) d t\right) d T-R_{\mathrm{av}}\left(V_{\mathrm{lv}}\left(t_{e}\right)-V_{\mathrm{lv}}(T)\right)
\end{aligned}
$$

where $T \in\left[t_{e}, t_{e}+\tau_{e}\right]$, and where the terms inside the integrals $\left(Q_{\mathrm{av}}, Q_{\mathrm{sys}}\right.$, $\left.V_{\text {lvf }}, P_{\text {peri }}\right)$ are measured or reasonably estimated, as discussed previously.

Similarly, integrating Equation (35) from $t_{f}$ to $T^{\prime}$ yields:

$$
\begin{aligned}
L_{\mathrm{mt}}\left(Q_{\mathrm{mt}}\left(T^{\prime}\right)-Q_{\mathrm{mt}}\left(t_{f}\right)\right) & =P_{\mathrm{pu} 0}\left(T^{\prime}-t_{f}\right)+E_{\mathrm{pu}} \int_{t_{f}}^{T^{\prime}}\left(\int_{0}^{t}\left(Q_{\mathrm{pul}}-Q_{\mathrm{mt}}\right) d t\right) d T^{\prime} \\
& +\int_{t_{f}}^{T^{\prime}}\left(P_{\mathrm{th}}-P_{\mathrm{peri}}\right)-E_{\mathrm{es}, \mathrm{lvf}} \int_{t_{f}}^{T^{\prime}} e V_{\mathrm{lvf}} d T^{\prime} \\
& -P_{0, \mathrm{lvf}} \int_{t_{f}}^{T^{\prime}}(1-e)\left(e^{\lambda_{\mathrm{lvf}} V_{\mathrm{lvf}}}-1\right) d T^{\prime}-R_{\mathrm{mt}}\left(V_{\mathrm{lv}}\left(T^{\prime}\right)-V_{\mathrm{lv}}\left(t_{f}\right)\right)
\end{aligned}
$$

where $T^{\prime} \in\left[t_{f}, t_{f}+\tau_{f}\right]$, and $\tau_{e}$ and $\tau_{f}$ are the times for ejection and filling of the left ventricle. 
Note that in practice the integral sign corresponds to numerical integration, which effectively acts as a low pass filter, so the effect of any noise in the flow measurements is significantly reduced. Finally, Equations (34)-(37) consider only the left ventricle and the aortic (ao) and pulmonary (pu) chambers of Figure 3. The right ventricle and the vena cava (vc) and pulmonary artery (pa) chambers can be treated exactly the same.

\subsection{Matrix system}

Equations (36) and (37) can now be written as a matrix system where there are more equations than unknowns, thus there is a unique linear least squares solution. More specifically, taking $P_{\text {th }}=-4 \mathrm{~mm} \mathrm{Hg}[1 ; 3 ; 18]$ and assuming $V_{\mathrm{spt}}=0 \mathrm{ml}$ or approximated, $V_{\mathrm{lvf}}$ can be found from Equation (18). Therefore all the integrals involve quantities that are either given or determined from measurement.

Thus, by choosing various values of $T$ during the ejection period and $T^{\prime}$ during the filling period of the left ventricle a set of linear equations can be set up in the 8 unknown parameters $L_{\mathrm{av}}, L_{\mathrm{mt}}, E_{\mathrm{es}, \mathrm{lvf}}, P_{0, \mathrm{lvf}}, R_{\mathrm{av}}, R_{\mathrm{mt}}, P_{\mathrm{ao} 0}$, $P_{\mathrm{pu} 0}$. If inertial effects are ignored $L_{\mathrm{mt}}$ and $L_{\mathrm{av}}$ can be ignored.

After applying the same method to the right ventricle, a set of $N$ linear equations can be set up in 16 variables to identify the patient specific parameters in the full model of Figure 3 . The variable vector, $\beta$, is defined in two sets:

$$
\underline{\beta}=\left[\underline{\alpha}, P_{\mathrm{ao} 0}, P_{\mathrm{pu} 0}, P_{\mathrm{pa} 0}, P_{\mathrm{vc} 0}\right],
$$


where:

$\underline{\alpha}=\left[L_{\mathrm{av}}, L_{\mathrm{mt}}, L_{\mathrm{tc}}, L_{\mathrm{pv}}, E_{\mathrm{es}, \mathrm{lvf}}, P_{0, \mathrm{lvf}}, E_{\mathrm{es}, \mathrm{rvf}}, P_{0, \mathrm{rvf}}, R_{\mathrm{av}}, R_{\mathrm{mt}}, R_{\mathrm{tc}}, R_{\mathrm{pv}}\right] \in \mathbf{R}^{16 \times 1}$

The last four variables in Equation $(38) P_{\mathrm{ao} 0}, P_{\mathrm{pu} 0}, P_{\mathrm{pa} 0}, P_{\mathrm{vc} 0}$ included as in Equations (32)-(33) to keep the equations linear in the unknowns. The patient specific parameters to be identified for the full model are given by $\underline{\alpha}$ in Equation (39). Finally, for clarity the estimated and measured variables are defined by $\underline{\gamma}$.

$$
\underline{\gamma}=\left[Q_{\mathrm{av}}, Q_{\mathrm{mt}}, Q_{\mathrm{tc}}, Q_{\mathrm{pv}}, Q_{\mathrm{sys}}, Q_{\mathrm{pul}}, P_{\mathrm{ao}}, P_{\mathrm{pa}}, V_{\mathrm{lv}}, V_{\mathrm{rv}}, V_{\mathrm{lvf}}, V_{\mathrm{rvf}}, P_{\mathrm{peri}}\right]
$$

In critical care these values can be obtained by a variety of means including ultrasound and echo-cardiography (e.g. [9]-[17]), or estimated.

For $n_{1}$ values of $T=T_{i} \in\left[t_{e}, t_{e}=\tau_{e}\right]$ and $n_{2}$ values of $T^{\prime}=T_{j}^{\prime} \in$ $\left[t_{f}, t_{f}+\tau_{f}\right]$, Equations (36) and (37) can be numerically integrated and written as a matrix system:

$$
A_{\mathrm{lv}} \underline{\beta}=b_{\mathrm{lv}}
$$

where the $i^{\text {th }}$ row of the $\left(n_{1}+n_{2}\right) \times 16$ matrix $A_{\mathrm{lv}}$ is defined:

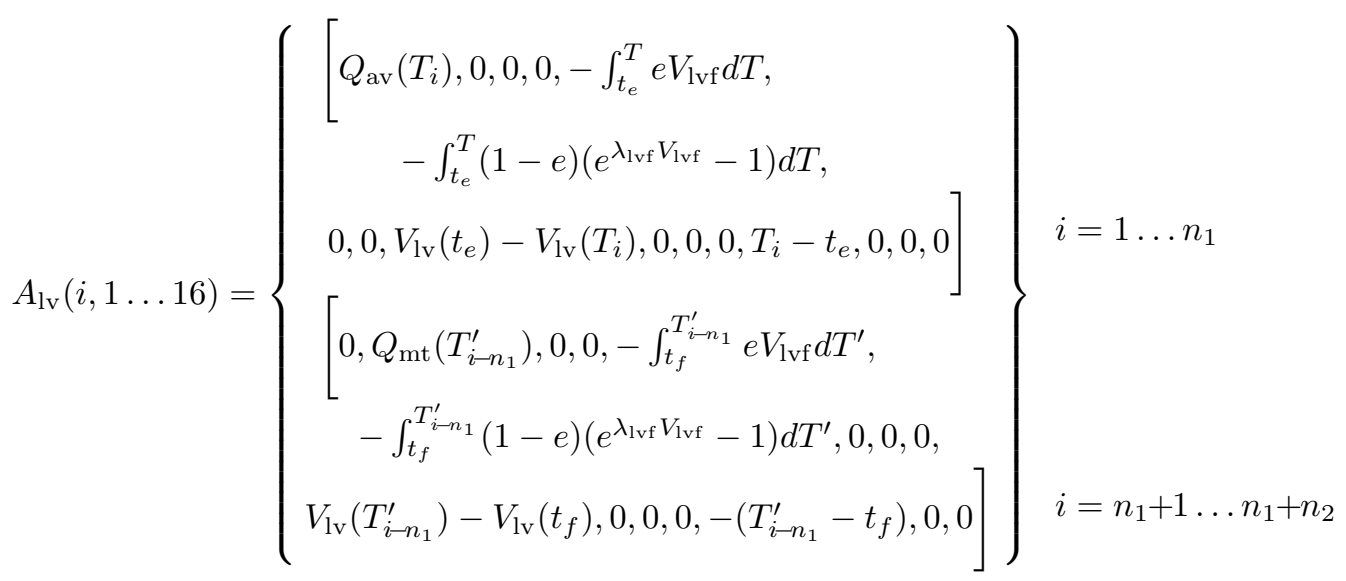


and the $\left(n_{1}+n_{2} \times 1\right)$ vector $b_{\mathrm{lv}}$ is defined:

$$
b_{\mathrm{lv}}=\left[b_{\mathrm{lv}, 1}, b_{\mathrm{lv}, 2}\right]^{T}
$$

where $b_{\mathrm{lv}, 1}$ is a $\left(1 \times n_{1}\right)$ vector and $b_{\mathrm{lv}, 2}$ is a $\left(1 \times n_{2}\right)$ vector defined:

$$
\begin{aligned}
b_{\mathrm{lv}, 1}= & {\left[\int_{t_{e}}^{T_{1}} P_{\mathrm{peri}} d T-E_{\mathrm{ao}} \int_{t_{e}}^{T_{1}}\left(\int_{0}^{t}\left(Q_{\mathrm{av}}-Q_{\mathrm{sys}}\right) d t\right) d T, \ldots,\right.} \\
b_{\mathrm{lv}, 2}= & {\left[\int_{t_{f}}^{T_{n_{1}}} P_{\mathrm{peri}} d T-E_{\mathrm{ao}} \int_{t_{e}}^{T_{1}^{\prime}}\left(P_{\mathrm{th}}-P_{\mathrm{peri}}\right) d T^{\prime}+E_{\mathrm{pu}}\left(Q_{t_{f}}^{T_{1}^{\prime}}\left(\int_{0}^{t}\left(Q_{\mathrm{pul}}-Q_{\mathrm{sys}}\right) d t\right) d T\right]\right.} \\
& \left.\left.\int_{t_{f}}^{T_{n_{2}}^{\prime}}\left(P_{\mathrm{th}}-P_{\mathrm{peri}}\right) d t\right) d T^{\prime}+E_{\mathrm{pu}} \int_{t_{f}}^{T_{n_{2}}^{\prime}}\left(\int_{0}^{t}\left(Q_{\mathrm{pul}}-Q_{\mathrm{mt}}\right) d t\right) d T^{\prime}\right]
\end{aligned}
$$

Note that $Q_{\mathrm{av}}\left(t_{e}\right)=0$ and $Q_{\mathrm{mt}}\left(t_{f}\right)=0$ as illustrated in Figure 2 .

An $\left(N_{1}+N_{2}\right) \times 16$ matrix $A_{\mathrm{rv}}$ and an $\left(N_{1}+N_{2}\right) \times 1$ vector $b_{\mathrm{rv}}$ can be set up for the right ventricle in a similar way where $N_{1}$ and $N_{2}$ are the number of time values chosen in the filling and ejection periods of the right ventricle. The matrices and vectors for the left and right ventricles can be combined to complete the matrix system relating all 16 parameters.

$$
A \underline{\beta}=b .
$$

where:

$$
A=\left(\begin{array}{c}
A_{\mathrm{lv}} \\
A_{\mathrm{rv}}
\end{array}\right), \quad b=\left(\begin{array}{c}
b_{\mathrm{lv}} \\
b_{\mathrm{rv}}
\end{array}\right) .
$$

Thus Equation (46) defines a set of $N=n_{1}+n_{2}+N_{1}+N_{2}$ equations in 16 unknowns.

Therefore, to calculate the numerical values in $A$ and $b$ requires the measured flows $Q_{\mathrm{av}}, Q_{\mathrm{mt}}, Q_{\mathrm{tc}}, Q_{\mathrm{pv}}$, the measured pressure waveforms $P_{\mathrm{ao}}, P_{\mathrm{pa}}$, the 
measured maximum and minimum volumes in each ventricle, the approximation to $V_{\mathrm{spt}}$ and assumed generic values of $\lambda_{\mathrm{lvf}}, \lambda_{\mathrm{rvf}}, P_{\mathrm{th}}, P_{0, \mathrm{pcd}}, \lambda_{\mathrm{pcd}}$ and $V_{0, \text { pcd }}$ defined in [3].

For Equations (42)-(43) of the left ventricle, assuming $V_{\mathrm{spt}}=0 \mathrm{ml}$, or approximated, $e(t), V_{\mathrm{lv}}$ and $V_{\mathrm{lvf}}$ are given by Equations (5), (10) and (18). Similarly, given generic values of $\lambda_{\mathrm{lv}}, P_{\mathrm{th}}, P_{0, \text { pcd }}, \lambda_{\mathrm{pcd}}$ and $V_{0, \mathrm{pcd}}, P_{\mathrm{peri}}$ is determined by Equations (19)-(21). The flows $Q_{\mathrm{av}}$ and $Q_{\mathrm{mt}}$ can be measured and the systemic and pulmonary flows $Q_{\text {sys }}$ and $Q_{\text {pul }}$ and the parameters $E_{\text {ao }}$ and $E_{\mathrm{pu}}$ are approximated. All integrals are numerically evaluated using the trapezium rule.

\subsection{Determining $Q_{\text {sys }}$ and $Q_{\text {pul }}$}

The goal is to determine the patient parameters $E_{\mathrm{ao}}, E_{\mathrm{pa}}, E_{\mathrm{vc}}, E_{\mathrm{pu}}, R_{\mathrm{sys}}, R_{\mathrm{pul}}$ and the flows $Q_{\text {sys }}$ and $Q_{\text {pul }}$ so that the integrals in Equations (42)-(47) can be numerically evaluated and used to set up the matrix $\bar{A}$ and vector $\bar{b}$ defined by Equations (67) and (68). The measured data required in this case is the pressure waveforms through the aorta $\left(P_{\mathrm{ao}}\right)$ and pulmonary artery $\left(P_{\mathrm{pa}}\right)$ in Figure 3 , and the flows $\left(Q_{\mathrm{av}}, Q_{\mathrm{mt}}\right.$ and $\left.Q_{\mathrm{tc}}, Q_{\mathrm{pv}}\right)$ into and out of the left and right ventricles.

The pressures in the vena cava $\left(P_{\mathrm{vc}}\right)$ and pulmonary vein $\left(P_{\mathrm{pu}}\right)$ are close to constant when compared to the pressure in the aorta $\left(P_{\mathrm{ao}}\right)$ and pulmonary artery $\left(P_{\mathrm{pa}}\right),[2 ; 3]$. Figure 4 shows all these pressures for the mitral stenosis case as an example. Thus, assuming $P_{\mathrm{vc}}=P_{\mathrm{vc} 0}$ and $P_{\mathrm{pu}}=P_{\mathrm{pu} 0}$ are constant, Equation (6) can be substituted into Equation (30) and solved for $P_{\text {ao }}$ to give 
a new expression for the pressure in the aorta,

$$
\begin{aligned}
\bar{P}_{\mathrm{ao}} & =P_{\mathrm{ao} 0}+E_{\mathrm{ao}} \int_{0}^{t} Q_{\mathrm{av}} d t-\frac{E_{\mathrm{ao}}}{R_{\mathrm{sys}}} \int_{0}^{t} P_{\mathrm{ao}} d t+\frac{E_{\mathrm{ao}}}{R_{\mathrm{sys}}} \int_{0}^{t} P_{\mathrm{vc} 0} d t \\
& =P_{\mathrm{ao} 0}+E_{\mathrm{ao}} \int_{0}^{t} Q_{\mathrm{av}} d t+A_{1} \int_{0}^{t} P_{\mathrm{ao}} d t+A_{2} t
\end{aligned}
$$

where $A_{1}$ and $A_{2}$ are defined:

$$
A_{1}=-\frac{E_{\mathrm{ao}}}{R_{\mathrm{sys}}}, \quad A_{2}=\frac{E_{\mathrm{ao}} P_{\mathrm{vc} 0}}{R_{\mathrm{sys}}}
$$

Similarly, from the equations:

$$
\begin{aligned}
& \dot{V}_{\mathrm{pa}}=Q_{\mathrm{pv}}-Q_{\mathrm{pul}} \\
& P_{\mathrm{pa}}=E_{\mathrm{pa}} V_{\mathrm{pa}}+P_{\mathrm{th}}
\end{aligned}
$$

where $Q_{\mathrm{pv}}$ is the flow out of the right ventricle through the pulmonary valve, a new expression for the pressure in the pulmonary artery can be written,

$$
\bar{P}_{\mathrm{pa}}=P_{\mathrm{pa} 0}+E_{\mathrm{pa}} \int_{0}^{t} Q_{\mathrm{pv}} d t+B_{1} \int_{0}^{t} P_{\mathrm{pa}}+B_{2} t
$$

where $B_{1}$ and $B_{2}$ are defined:

$$
B_{1}=-\frac{E_{\mathrm{pa}}}{R_{\mathrm{pul}}}, \quad B_{2}=\frac{E_{\mathrm{pa}} P_{\mathrm{pu} 0}}{R_{\mathrm{pul}}}
$$

The integrals in Equations (48) and (51) are calculated numerically from measured data. The best linear least squares fit of $\bar{P}_{\mathrm{ao}}$ and $\bar{P}_{\mathrm{pa}}$ to the measured waveforms of $P_{\mathrm{ao}}$ and $P_{\mathrm{pa}}$ will give an approximation to the parameters $E_{\text {ao }}$ and $E_{\text {pa }}$. Finally, rearranging Equation (30) yields:

$$
\int_{0}^{t} Q_{\mathrm{sys}}=\frac{1}{E_{\mathrm{ao}}}\left(P_{\mathrm{ao} 0}+E_{\mathrm{ao}} \int_{0}^{t} Q_{\mathrm{av}}-P_{\mathrm{ao}}\right) .
$$


Thus, given the least squares best fit curve $\bar{P}_{\text {ao }}$ through $P_{\text {ao }}$ and the approximation of $E_{\mathrm{ao}}$, the value of $Q_{\mathrm{sys}}$ is estimated:

$$
\bar{Q}_{\mathrm{sys}}=\frac{1}{E_{\mathrm{ao}}}\left(\bar{P}_{\mathrm{ao}}(0)+E_{\mathrm{ao}} \int_{0}^{t} Q_{\mathrm{av}}-\bar{P}_{\mathrm{ao}}\right)^{\prime}
$$

Similarly, $Q_{\mathrm{pul}}$ is estimated:

$$
\bar{Q}_{\mathrm{pul}}=\frac{1}{E_{\mathrm{pa}}}\left(\bar{P}_{\mathrm{pa}}(0)+E_{\mathrm{pa}} \int_{0}^{t} Q_{\mathrm{pv}}-\bar{P}_{\mathrm{pa}}\right)^{\prime} .
$$

Integrating the equation:

$$
\dot{V}_{\mathrm{vc}}=\bar{Q}_{\mathrm{sys}}-Q_{\mathrm{tc}}
$$

where $V_{\mathrm{vc}}$ is the volume of the vena cava and $Q_{\mathrm{tc}}$ is the flow into the right ventricle through the tricuspid valve yields:

$$
V_{\mathrm{vc}}=V_{\mathrm{vc}}(0)+\int_{0}^{t}\left(\bar{Q}_{\mathrm{sys}}-Q_{\mathrm{tc}}\right) d t
$$

Using Equation (52), Equation (56) can be rewritten to determine the change in $V_{\mathrm{vc}}$.

$$
\Delta V_{\mathrm{vc}}=V_{\mathrm{vc}}-V_{\mathrm{vc}}(0)=\frac{1}{E_{\mathrm{ao}}}\left(\bar{P}_{\mathrm{ao} 0}+E_{\mathrm{ao}} \int_{0}^{t} Q_{\mathrm{av}}-\bar{P}_{\mathrm{ao}}\right)-\int_{0}^{t} Q_{\mathrm{tc}} d t .
$$

Multiplying Equation (6) by $R_{\text {sys }}$ then integrating gives:

$$
\int_{0}^{t} P_{\mathrm{ao}} d t=R_{\mathrm{sys}} I_{\mathrm{Q}_{\mathrm{sys}}}(t)+\int_{0}^{t} P_{\mathrm{vc}} d t
$$

where from Equation (52) a function $I_{\mathrm{Q}_{\mathrm{sys}}}(t)$ representing the integrated systemic flow $Q_{\text {sys }}$ can be defined using the approximated value $\bar{P}_{\text {ao }}$.

$$
I_{\mathrm{Q}_{\mathrm{sys}}}(t)=\frac{1}{E_{\mathrm{ao}}}\left(\bar{P}_{\mathrm{ao}}(0)+E_{\mathrm{ao}} \int_{0}^{t} Q_{\mathrm{av}} d t-\bar{P}_{\mathrm{ao}}\right)
$$


Equation (59) is clearly a function of $t$. Substituting $V_{\mathrm{vc}}=V_{\mathrm{vc}}(0)+\Delta V_{\mathrm{vc}}$ and Equation (26) into Equation (58) gives:

$$
\int_{0}^{t} P_{\mathrm{ao}} d t=R_{\mathrm{sys}} I_{\mathrm{Q}_{\mathrm{sys}}}(t)+E_{\mathrm{vc}} \int_{0}^{t} \Delta V_{\mathrm{vc}} d t+E_{\mathrm{vc}} V_{\mathrm{vc}}(0) t
$$

where from Equation (57), $\Delta V_{\mathrm{vc}}$ is a known function. Defining,

$$
I_{\mathrm{P}_{\mathrm{ao}}}(t)=R_{\mathrm{sys}} I_{\mathrm{Q}_{\mathrm{sys}}}(t)+E_{\mathrm{vc}} \int_{0}^{t} \Delta V_{\mathrm{vc}} d t+a_{\mathrm{P}_{\mathrm{ao}}} t
$$

where,

$$
a_{\mathrm{P}_{\mathrm{ao}}}=E_{\mathrm{vc}} V_{\mathrm{vc}}(0)
$$

and finding the best least squares fit of $I_{\mathrm{P}_{\mathrm{ao}}}(t)$ to $\int_{0}^{t} \bar{P}_{\mathrm{ao}} d t$ will yield an estimate of $R_{\mathrm{sys}}$ and $E_{\mathrm{vc}}$. In a similar way, the parameters $R_{\mathrm{pul}}$ and $E_{\mathrm{pu}}$ can be determined from the measured waveform $P_{\mathrm{pa}}$.

In summary, the parameters $E_{\mathrm{ao}}, E_{\mathrm{pa}}, E_{\mathrm{vc}}, E_{\mathrm{pu}}, R_{\mathrm{sys}}, R_{\mathrm{pul}}$ and the flows through the systemic and pulmonary circulations, $Q_{\text {sys }}$ and $Q_{\text {pul }}$ are determined from the measured pressure waveforms through the aorta and pulmonary artery and the flows into and out of each ventricle.

\subsection{Alternative formulation}

The inertances are difficult to measure and represent the inertia of blood volumes and are not well defined [3]. Hence, solving Equation (46) with all the parameters $\underline{\beta}$ at once may require physiological constraints on the values of $L_{\mathrm{av}}, L_{\mathrm{mt}}, L_{\mathrm{pv}}$ and $L_{\mathrm{tc}}$, which will take longer to solve using constrained linear least squares and be potentially less accurate. In addition, $L_{\mathrm{av}}, L_{\mathrm{mt}}$, $L_{\mathrm{pv}}$ and $L_{\mathrm{tc}}$ are of the order $10^{-2}$ smaller than the rest of the parameters and 
are therefore prone to numerical error in solution. The alternative formulation presented manages this problem by identifying $L_{\mathrm{av}}, L_{\mathrm{mt}}, L_{\mathrm{pv}}$ and $L_{\mathrm{tc}}$, are separately.

For a given set of inertances $L_{\mathrm{av}}, L_{\mathrm{mt}}, L_{\mathrm{tc}}$ and $L_{\mathrm{pv}}$ an alternative set of $N$ linear equations can be set up in 12 variables $\underline{\beta}(5 \ldots 16)$. Dividing Equation (36) by $L_{\mathrm{av}}$ and Equation (37) by $L_{\mathrm{mt}}$ gives the matrix system:

$$
\bar{A}_{\mathrm{lv}}(\underline{\beta}(5 \ldots 16))=\bar{b}_{\mathrm{lv}}
$$

where $\bar{A}_{\mathrm{lv}}$ is an $\left(n_{1}+n_{2}\right) \times 12$ matrix and is defined:

$$
\bar{A}_{\mathrm{lv}}=\left(\begin{array}{c}
\frac{1}{L_{\mathrm{av}}} A_{\mathrm{lv}}\left(5 \ldots 16,1 \ldots n_{1}\right) \\
\frac{1}{L_{\mathrm{mt}}} A_{\mathrm{lv}}\left(5 \ldots 16, n_{1}+1 \ldots n_{1}+n_{2}\right)
\end{array}\right)
$$

and $\bar{b}_{\mathrm{lv}}$ is an $\left(n_{1}+n_{2}\right) \times 1$ vector defined:

$$
\bar{b}_{\mathrm{lv}}=\left(\begin{array}{c}
\frac{1}{L_{\mathrm{av}}} b_{\mathrm{lv}}\left(1 \ldots n_{1}\right)-\left[Q_{\mathrm{av}}\left(T_{1}\right), \ldots, Q_{\mathrm{av}}\left(T_{n_{1}}\right)\right]^{T} \\
\frac{1}{L_{\mathrm{mt}}} b_{\mathrm{lv}}\left(n_{1}+1, \ldots, n_{1}+n_{2}\right)-\left[Q_{\mathrm{mt}}\left(T_{1}^{\prime}\right), \ldots, Q_{\mathrm{mt}}\left(T_{n_{2}}^{\prime}\right)\right]^{T}
\end{array}\right)
$$

Similarly, an $\left(N_{1}+N_{2}\right) \times 12$ matrix $\bar{A}_{\text {rv }}$ and an $\left(N_{1}+N_{2}\right) \times 1$ vector $\bar{b}_{\text {rv }}$ can be formed for the right ventricle. Thus, the resulting set of $N=n_{1}+n_{2}+N_{1}+N_{2}$ equations in 12 unknowns $\underline{\beta}(5 \ldots 16)$ can be written as a matrix system.

$$
\bar{A}(\underline{\beta}(5 \ldots 16))=\bar{b}
$$

where $\bar{A}$ and $\bar{b}$ are defined as functions of the inertance values.

$$
\begin{gathered}
\bar{A}=\bar{A}\left(L_{\mathrm{av}}, L_{\mathrm{mt}}, L_{\mathrm{tc}}, L_{\mathrm{pv}}\right)=\left(\begin{array}{c}
\bar{A}_{\mathrm{lv}} \\
\bar{A}_{\mathrm{rv}}
\end{array}\right) \\
\bar{b}=\bar{b}\left(L_{\mathrm{av}}, L_{\mathrm{mt}}, L_{\mathrm{tc}}, L_{\mathrm{pv}}\right)=\left(\begin{array}{c}
\bar{b}_{\mathrm{lv}} \\
\bar{b}_{\mathrm{rv}}
\end{array}\right)
\end{gathered}
$$


Hence, for given values of $L_{\mathrm{av}}, L_{\mathrm{mt}}, L_{\mathrm{tc}}$ and $L_{\mathrm{pv}}$ the rest of the parameters are given by the unique linear least squares solution to Equation (66). Therefore, if the function $F\left(L_{\mathrm{av}}, L_{\mathrm{mt}}, L_{\mathrm{tc}}, L_{\mathrm{pv}}\right)$ is defined:

$$
F\left(L_{\mathrm{av}}, L_{\mathrm{mt}}, L_{\mathrm{tc}}, L_{\mathrm{pv}}\right)=\bar{A}(\underline{\beta}(5 \ldots 16))-\bar{b}
$$

then $\|F\|_{2}$ has a minimum value at the true values of $L_{\mathrm{av}}, L_{\mathrm{mt}}, L_{\mathrm{tc}}$ and $L_{\mathrm{pv}}$, and the original problem can be simplified. These inertances and the rest of the parameters are therefore found by minimizing the function $F$. Note that each function evaluation of $F$ involves solving Equation (66) by linear least squares. Note also that the sum of the initial volumes:

$$
V_{\mathrm{ao} 0}+V_{\mathrm{pu} 0}+V_{\mathrm{pa} 0}+V_{\mathrm{vc} 0}+V_{\mathrm{lv}}(0)+V_{\mathrm{rv}}(0)=1500
$$

where

$$
V_{\mathrm{ao} 0}=\frac{P_{\mathrm{ao}}}{E_{\mathrm{ao}}}, \quad V_{\mathrm{pu} 0}=\frac{P_{\mathrm{pu} 0}}{E_{\mathrm{pu}}}, \quad V_{\mathrm{pa} 0}=\frac{P_{\mathrm{pa} 0}}{E_{\mathrm{pa}}}, \quad V_{\mathrm{vc} 0}=\frac{P_{\mathrm{vc} 0}}{E_{\mathrm{vc}}}
$$

is constrained to be equal to $1500 \mathrm{ml}$, the total unstressed volume of blood assumed in this model $[1 ; 3]$. This constraint was added by including:

$$
V_{\mathrm{ao} 0}+V_{\mathrm{pu} 0}+V_{\mathrm{pa} 0}+V_{\mathrm{vc} 0}+V_{\mathrm{lv}}(0)+V_{\mathrm{rv}}(0)-1500
$$

as an extra equation in the vector function $F\left(L_{\mathrm{av}}, L_{\mathrm{mt}}, L_{\mathrm{pv}}, L_{\mathrm{tc}}\right)$ defined in Equation (69).

In summary,

- The parameters $\lambda_{\mathrm{lvf}}, \lambda_{\mathrm{rvf}}, P_{\mathrm{th}}, P_{0, \mathrm{pcd}}, \lambda_{\mathrm{pcd}}, V_{0, \mathrm{pcd}}, E_{\mathrm{ao}}, E_{\mathrm{pa}}, E_{\mathrm{pu}}, E_{\mathrm{vc}}, R_{\mathrm{sys}}$ are either fixed at generic values or approximated using measured data 
- The time varying dependent parameters $e(t)$ and $\underline{\gamma}$ in Equation (40), are either given, measured or determined from measurement

- The numerical values of the matrix $A$ and vector $b$ of Equation (47) which involve the Equations (42) and (43) of the left ventricle and similar equations for the right ventricle are calculated using numerical integration

- The matrix $\bar{A}$ and vector $\bar{b}$ given by Equations (64)-(68) are determined for a given set of inertances $L_{\mathrm{av}}, L_{\mathrm{mt}}, L_{\mathrm{tc}}$ and $L_{\mathrm{pv}}$ using the numerical values of the matrix $A$ and vector $b$ of Equation (47)

- The unknown patient specific parameters are defined in Equations (38) and (39) and are found by minimizing the function $F$ defined in Equation (69).

\section{Results}

A healthy human is simulated first, using the full model with outputs shown in Table 1. These results agree well with values reported in medical physiology texts for example [18]. The resistances used in simulating the healthy human are shown in the first column of Table 2 .

Valvular Stenosis is caused by calcium deposition on the surface of a valve, which limits the valve's ability to open properly. The resistance $R_{\mathrm{mt}}$ of the mitral valve is doubled to simulate mitral stenosis [3]. The average pressure in the pulmonary vein rises from $P_{\mathrm{pu}}=2 \mathrm{mmHg}$ to $3.5 \mathrm{mmHg}$, the average 
left ventricle volume decreases from $76.3 \mathrm{ml}$ to $66.6 \mathrm{ml}$ and the stroke volume decreases from $66.0 \mathrm{ml}$ to $59.0 \mathrm{ml}$, matching known physiological trends [19].

Pulmonary Embolism is caused by obstruction to the blood flow in the pulmonary circulation system, commonly a blood clot. The pulmonary vas-

cular resistance $R_{\text {pul }}$ is increased by a factor of two to simulate Pulmonary Embolism. Cardiac output drops from $5.3 \mathrm{~L} / \mathrm{min}$ to $4.8 \mathrm{~L} / \mathrm{min}$, the maximum pressure in the pulmonary artery increases from $25.7 \mathrm{mmHg}$ to $35.4 \mathrm{mmHg}$ and the average pressure in the vena cava increases from $2.0 \mathrm{mmHg}$ to $2.4 \mathrm{mmHg}$, also matching known trends [19].

The flows around the cardiac chambers (i.e in and out of each ventricle) and the pressure waveforms through the aorta and pulmonary artery are discretized for each simulation, analogous to measured data. Figures 6 and 7 show the flow through the aortic and mitral valves for a healthy human and the two disease states. As noted, these flows can be measured by echocardiography. Equally importantly, these "measured" values represent a very limited set of measurements compared to the full set of model pressures, volumes and flows. The results of each simulation are discretized and used to test the identification method presented. Accuracy is measured by comparing identified parameters to the "true" values used in the original simulation.

\subsection{Implementation of the integral method}

The alternative formulation of the integral method of Equations (64)-(68) is first applied to identify the patient specific parameters for the healthy human. However, the volume of the septum $V_{\mathrm{spt}}$ is unknown and not directly 
measurable. As an initial approximation, it is set to zero $\left(V_{\mathrm{spt}}=0\right)$. The resulting optimized parameters from the integral method are then used to rerun the model and obtain a significantly better approximation to $V_{\mathrm{spt}}$. Figure 5 shows the approximated $V_{\text {spt }}$ versus the true, simulated $V_{\text {spt }}$ from this step. The integral method is then applied a second time with the approximated $V_{\text {spt }}$ value producing the results shown in Table 2. Note that this approach requires a second identification but this includes this last model variable $\left(V_{\mathrm{spt}}\right)$.

A similar method was applied for Mitral Stenosis and Pulmonary Embolism. Table 2 shows that $R_{\mathrm{mt}}$ and $R_{\mathrm{pul}}$ are identified accurately in the healthy human and each disease state with less than $1 \%$ error. Importantly, the other identified parameters in all three cases were unchanged from the "true" values used to generate the simulated data and thus accurately identified. Therefore, these two disease states are accurately identified with the full model based only on measured flows, the maximum and minimum volumes of the left and right ventricles and the pressure waveforms through the aorta and pulmonary artery.

All measured data simulated in this research can be obtained non-invasively using echo-cardiography and ultra-sound. Using the identified patient specific parameters the model can be rerun to obtain the model response error for the output flows. The mean percentage error in total is $0.15 \%$ with a standard deviation of $0.17 \%$ further validating the integral method developed. Figure 8 is an example of the flow output through the mitral valve with optimized values (solid line) versus the true "measured" values (circles) for mitral stenosis, showing a very close match. 


\section{$3.2 \quad$ Impact of noise}

In practical situations, measurements will not be free of noise. In this case, 5, 10 and $15 \%$ uniform distributed noise is added to the measured flow and the pressure waveforms through the aorta and pulmonary artery. A uniform distribution is used as a conservative choice where outliers are much more likely to occur.

Figure 9 shows the flow through the mitral valve with $5 \%$ noise for Mitral Stenosis. Table 3 shows that for all levels of noise the method successively identified the increased resistance of the mitral valve. Note that for $15 \%$ noise, the mean model response error in all the flows compared to the true flows was $0.9 \%$ with a standard deviation of $1.0 \%$ and the mean model response error of the pressures of the aorta and pulmonary artery were $1.0 \%$ and $4.5 \%$ with standard deviations of $0.4 \%$ and $2.6 \%$. Hence, the integral method is very effective in matching the data in the presence of significant measurement noise. Similar results were obtained for Pulmonary Embolism and are shown in Table 4.

\section{Discussion}

The integral based optimization successively identified patient specific parameters for the full six chamber minimal cardiac model with inertial effects and ventricular interaction using only measured cardiac chamber flows, the minimum and maximum volumes in the left and right ventricles and the pressure waveforms through the aorta and pulmonary artery. The use of integrals means measurement noise is low pass filtered, as demonstrated by 
the accurate matching of the identified model output to the original virtual patient results with up to $15 \%$ uniformly distributed noise.

Computationally, the parameter identification optimization problem is made linear and convex where current approaches are non-linear and nonconvex. In addition, the differential equations are not required to be solved each iteration and initial conditions or guesses for the parameter values are not needed. Thus, the issue of the incorrect initial conditions leading to increased time for model convergence, non-optimal or false results are avoided $[3]$.

Patient specific parameters for the healthy human and two disease states are accurately identified in the presence of significant noise with minimal computation, showing the method is applicable to a clinical setting. This integral approach ensures medical staff can obtain rapid patient specific information to assist in diagnosis and therapy selection in clinical real time (3-5 minutes). Hence, the reduction in computation enables its practical, clinical use in a decision support role.

\subsection{Limitations and possible extensions}

The underlying minimal model has been extensively verified with the trends of a significant variety of disease states and sensitivity analysis shows that most parameters have a unique localized impact [1]-[3]. However, because of assumptions in the model, for example holding the parameters in the pericardium at generic values and using a simple time varying elastance curve, there is always the possibility of the model missing dynamics that may exist 
clinically. Such errors would show up as an error in the data fitting. More specifically, while the model has been extensively verified using clinical data for both steady state and transient dynamics [1]-[3], there remains the possibility of unmodelled dynamics being observed clinically. Future work will involve more direct clinical verification of this model and methods.

The approach of this research is to model the primary parameters that govern the major dynamics of a patient's physiological state and only bring in extra parameters as required. The integral approach presented greatly simplifies the process of identifying these primary parameters. Hence, there is significantly more scope for introducing further complexity into the model to capture subtle behaviors that may not be represented in the model yet. Current non-linear regression methods [4] are severely limited in this regard.

For example, the parameters $E_{\mathrm{es}}$ and $P_{0}$ could be made piecewise constant over say $10-20$ equally spaced time intervals in a single heart beat to pick up any deviation in the currently assumed time varying elastance curve. In this case, the number of variables in the optimization will increase by the number of equally spaced time intervals, but the optimization will still be linear and convex using this parametrization.

The overall approach is to use a number of linear optimizations for each fixed value of the non-linear parameters rather than one large non-linear optimization of all the parameters. In the case of the current model, if the non-linear pericardium parameters $P_{0, \mathrm{pcd}}, \lambda_{\mathrm{pcd}}, V_{0, \mathrm{pcd}}$ are included there are $18+3=21$ patient parameters to be identified. The integral approach effectively reduces the number of parameters to this set of three pericardium parameters since the rest of the parameters can be first found by linear 
least squares optimization before identifying these remaining terms. Iteration between identification problems would then lead to convergence.

Current non-linear regression approaches would have to deal with all 21 parameters, as well as the large cost of solving the differential equations each time. Thus, to remain computationally viable in clinical real time, there is a severe restriction on the amount of extra complexity that can be added to the model. The integral based approach presented therefore allows a significantly greater flexibility in the modelling to deal with any potentially missing dynamics found clinically.

However, note that the parameters $E_{\mathrm{ao}}, E_{\mathrm{pa}}, E_{\mathrm{vc}}, E_{\mathrm{pu}}, R_{\mathrm{sys}}$ and $R_{\mathrm{pul}}$ can be found independently of all the rest of the parameters in the model, as they only depend on the measured flows into and out of each ventricle, and the measured pressure waveforms through the aorta and pulmonary artery. Thus, any dynamics of the flows that may not be captured in the current model because of, for example, a different time varying elastance curve, will not effect the calculation of the parameters $E_{\mathrm{ao}}, E_{\mathrm{pa}}, E_{\mathrm{vc}}, E_{\mathrm{pu}}, R_{\mathrm{sys}}$ and $R_{\mathrm{pul}}$. As these are the more critical parameters for identifying disease states [1]-[3], the overall approach should be robust. This result also indicates a much less complex way to solve some clinical problems.

\section{Conclusion}

An integral based optimization method is presented which turns a previously non-linear non-convex problem into a linear convex problem. Examples are given with a healthy human and two disease states to demonstrate 
the method. All parameters were identified successfully and the results for rerunning the model with the optimized parameters were very close to the original simulated outputs even with up to $15 \%$ uniformly distributed noise added.

Ventricular interaction was included by initially assuming the septum volume $V_{\mathrm{spt}}=0$ then rerunning the model with the approximate optimized parameters to obtain a better approximation. Apart from this one simulation the differential equations are not required to be solved and there is a unique linear least squares solution to the optimization equations. Note that if the pressure in the left and right ventricles is measured then this iteration is not needed as $V_{\mathrm{spt}}$ can be determined directly from $P_{\mathrm{lv}}-P_{\mathrm{rv}}$ [1]. The integral method significantly reduces the computation required and enables a fast and accurate method for identifying patient specific parameters. Also because of the speed of the method this allows much more scope for introducing further complexity into the model to capture more dynamics if required, for example adding an atrium. The non-linear method is severely restricted in this regard.

Clinically, this approach means patient specific parameters will be able to be found accurately and robustly using a standard modern desktop computer. Medical staff will be able to have rapid data on patients to assist in diagnosis and can trial and test therapies in clinical real time (3-5 minutes).

\section{References}

[1] B.W. Smith, J.G. Chase, R. I. Nokes, and G. Shaw, G.M.and Wake. Minimal haemodynamic system model including ventricular interaction 
and valve dynamics. Med. Eng. Phys, 26(2):131-139, 2004.

[2] B.W. Smith, J.G. Chase, G.M. Shaw, and R.I. Nokes. Experimentally verified minimal cardiovascular system model for rapid diagnostic assistance. Control Engineering Practice, in press, 2004.

[3] B.W. Smith. Minimal Haemodynamic Modelling of the Heart and Circulation for Clinical Application. Phd thesis, University of Canterbury, 2004.

[4] E. Carson and C Cobelli. Modelling Methodology for Physiology and Medicine. Academic Press, 2001.

[5] C.E. Hann, J.G. Chase, J. Lin, T. Lotz, C.V. Doran, and G.M. Shaw. Integral-based parameter identification for long-term dynamic verification of a glucose-insulin system model. J. of Computer Methods and Programs in Biomedicine, 2005, accepted 10/04 to appear.

[6] J. Lin, J.G. Chase, G.M. Shaw, T. Lotz, C.E. Hann, C.V. Doran, and D.S. Lee. Long term verification of glucose-insulin regulatory system model dynamics. Proc 26 th International Conf of IEEE Engineering in Med and Biology Society (EMBS 2004), San Francisco, CA, Sept 1-5, pages 758-761, 2004.

[7] R. Beyar, M.J. Hausknecht, H.R. Halperin, F.C. Yin, and M.L. Weisfeldt. Interaction between cardiac chambers and thoracic pressure in intact circulation. Am. J. Physiol, 22(253):H1240-52, 1987. 
[8] D. Chung. Ventricular interaction in a closed-loop model of the canine circulation. Master's thesis, Rice University, 1996.

[9] C.L. Moore, G.A. Rose, V.S. Tayal, and M. et al Sullivan. Determination of left ventricular function by emergency physician echocardiography of hypotensive patients. Academic Emergency Medicine, 2002.

[10] W.C. Shoemaker, A. Ayres, S. Grenvik, P.R. Holbrook, and W.L. Thompson. Textbook of Critical Care. W. B. Saunders Company, 1989.

[11] L. Moulinier, T. Venet, N.B Schiller, T.W. Kurtz, R.C. Jr Morris, and A. Sebastian. Measurement of aortic blood flow by doppler echocardiography: day to day variability in normal subjects and applicability in clinical research. J Am Coll Cardiol., 17(6):1326-1333, 1991.

[12] S.J. Goldberg, D.F. Dickinson, and N. Wilson. Evaluation of an elliptical area technique for calculating mitral blood flow by doppler echocardiography. British Heart Journal, 54:68-75, 1985.

[13] C.P. Appleton, L.K. Hatle, and R.L. Popp. Relation of transmitral flow velocity patterns to left ventricular diastolic function: new insights from a combined hemodynamic and doppler echocardiographic study. J Am Coll Cardiol, 12(2):426-440, 1988.

[14] J. Xu, L.G. Durand, and Pibarot P. A new, simple, and accurate method for noninvasive estimation of pulmonary arterial pressure. Heart, 88:7680, 2002.

[15] B. Popov, G. Sierra, L.G. Durand, J. Xu, P. Pibarot, R. Agarwal, and 
V. Lanzo. Automated extraction of aortic and pulmonary components of the second heart sound for the estimation of pulmonary artery pressure. Proceedings of the 26th Annual International conference of the IEEE Engineering in Medicine and Biology Society (EMBS 2004), San Francisco, CA, Sept 1-5, pages 921-924, 2004.

[16] K.L. Chan, P.J. Currie, J.B. Seward, D.J. Hagler, D.D. Mair, and A.J. Tajik. Comparison of three doppler ultrasound methods in the prediction of pulmonary artery pressure. J Am Coll Cardiol, 9(3):549-554, 1987.

[17] H. Watanabe, M. Kawai, T. Sibata, M. Hara, H. Furuhata, and S. Mochizuki. Noninvasive measurement of aortic pressure waveform by ultrasound. Heart Vessels, 13(2):79-86, 1998.

[18] A.C. Guyton and J.E. Hall. Textbook of Medical Physiology.

[19] E. Braunwald. Heart Disease, A text book of cardiovascular medicine, 5th edition. W.B. Saunders Company, Philadelphia, 1997. 


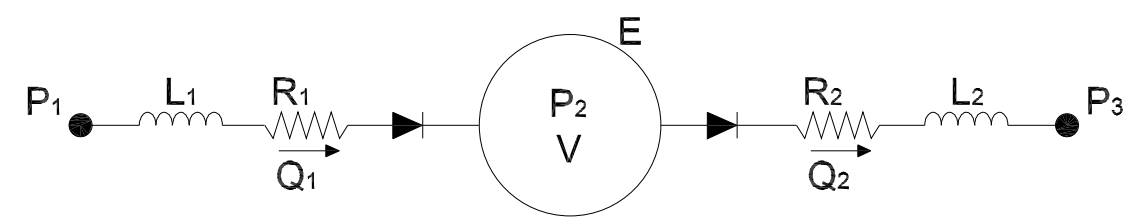

Figure 1: The single cardiac chamber model

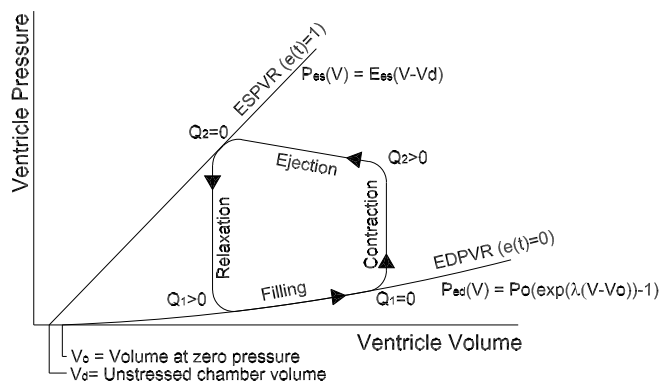

(a)

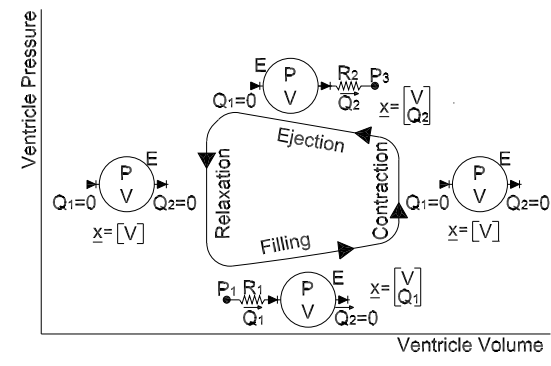

(b)

Figure 2: An example of a pressure-volume diagram with the different states of the single cardiac chamber model included. Right side shows model state at each part of the cardiac cycle.

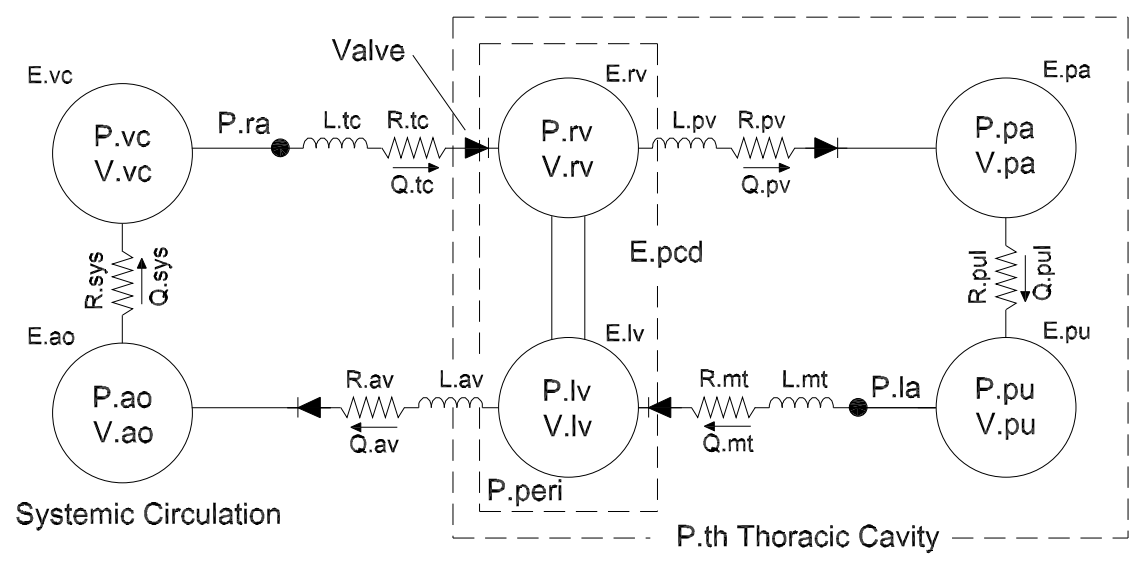

Figure 3: The presented closed loop model of the cardiovascular system. 


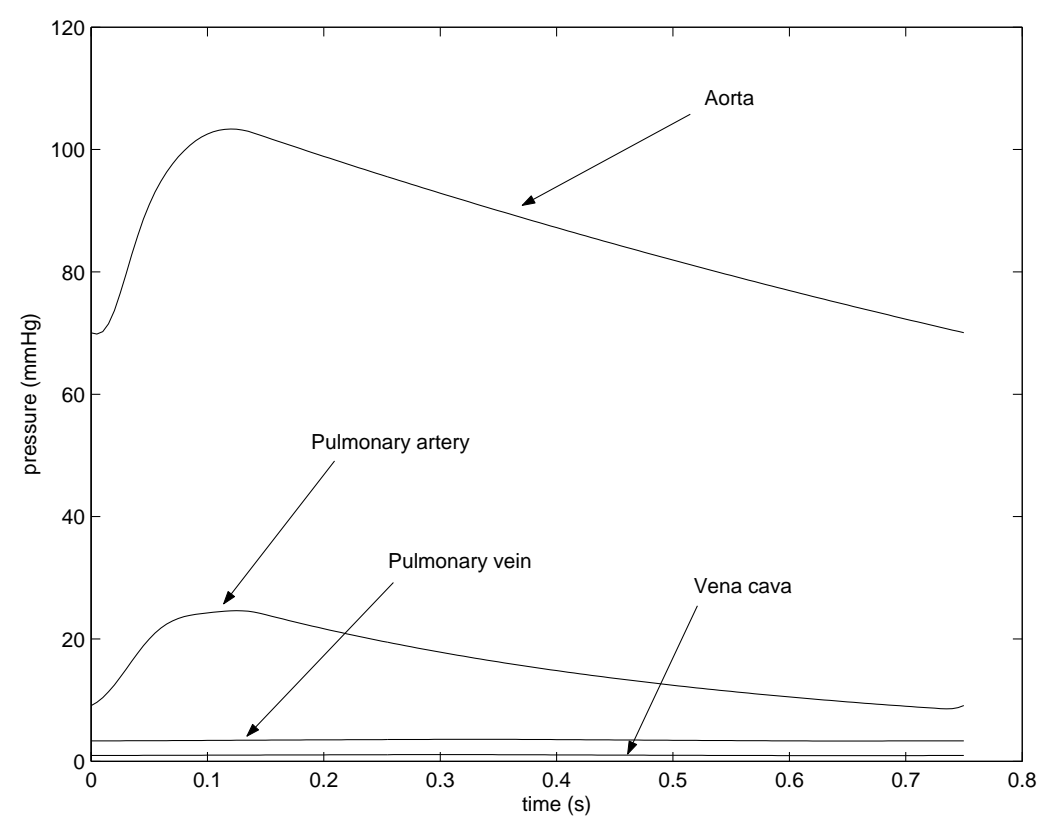

Figure 4: The pressures in the aorta and pulmonary artery compared to the pressures in the vena cava and the pulmonary vein for mitral stenosis.

\begin{tabular}{|l|l|}
\hline Description & Output \\
\hline Volume in left ventricle & $111.7 / 45.7 \mathrm{ml}$ \\
Volume in right ventricle & $112.2 / 46.1 \mathrm{ml}$ \\
Max $P_{\mathrm{lv}}$ & $119.2 \mathrm{mmHg}$ \\
Max $P_{\mathrm{rv}}$ & $26.2 \mathrm{mmHg}$ \\
Pressure in aorta & $116.6 / 79.1 \mathrm{mmHg}$ \\
Pressure in pulmonary artery & $25.7 / 7.8 \mathrm{mmHg}$ \\
Average pressure in pulmonary vein & $2.0 \mathrm{mmHg}$ \\
Average pressure in vena cava & $2.0 \mathrm{mmHg}$ \\
\hline
\end{tabular}

Table 1: Healthy human model outputs 


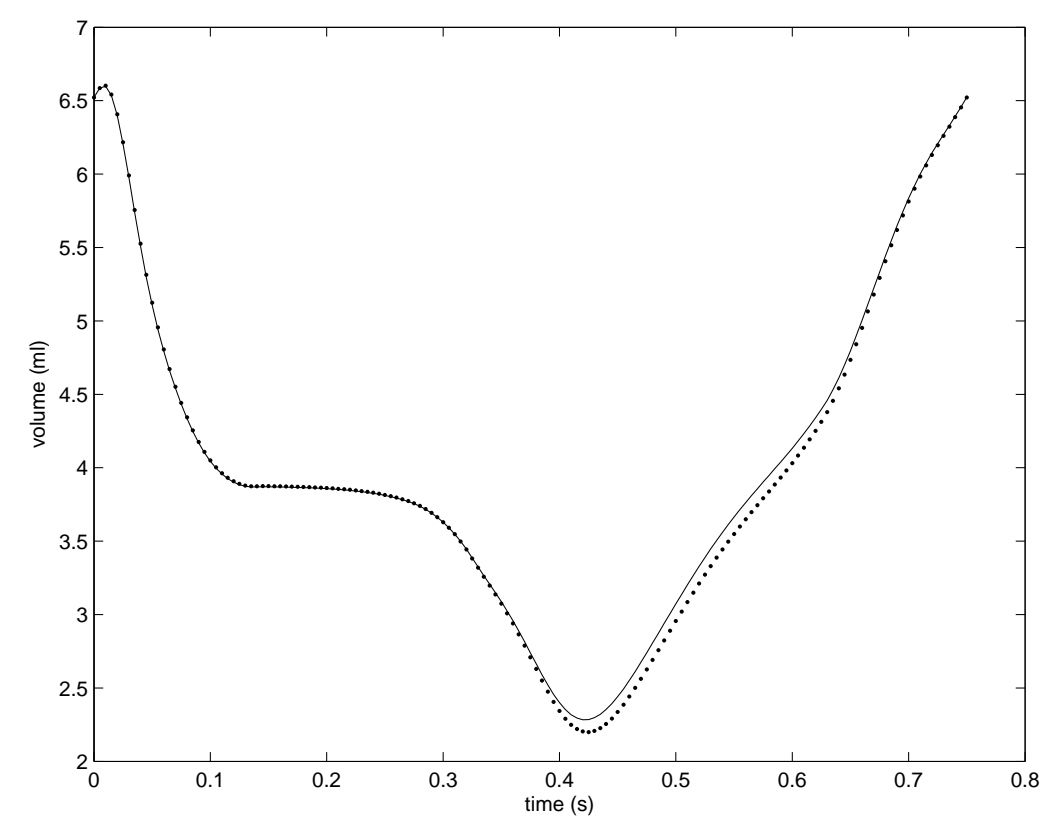

Figure 5: Approximated $V_{\text {spt }}$ (solid) versus true $V_{\text {spt }}$ (dots).

\begin{tabular}{|l|l|l|l|l|l|l|}
\hline & Healthy & Error (\%) & $\begin{array}{l}\text { Mitral } \\
\text { Stenosis }\end{array}$ & Error (\%) & $\begin{array}{l}\text { Pulmonary } \\
\text { Embolism }\end{array}$ & Error (\%) \\
\hline$R_{\mathrm{av}}($ Resistance) & 0.0180 & 0.06 & 0.0181 & 0.32 & 0.0183 & 1.89 \\
$R_{\mathrm{mt}}(\mathrm{mmHg} s / \mathrm{ml})$ & 0.0157 & 0.54 & 0.0315 & 0.10 & 0.0158 & 0.37 \\
$R_{\mathrm{pv}}$ & 0.0055 & 0.05 & 0.0055 & 0.80 & 0.0055 & 0.07 \\
$R_{\mathrm{tc}}$ & 0.0236 & 0.19 & 0.0235 & 0.90 & 0.0238 & 0.68 \\
$R_{\mathrm{sys}}$ & 1.0853 & 0.33 & 1.0888 & 0.01 & 1.1010 & 1.11 \\
$R_{\text {pul }}$ & 0.1552 & 0.001 & 0.1536 & 1.03 & 0.3099 & 0.16 \\
\hline $\begin{array}{l}\text { mean (including } \\
\text { all 18 parameters) }\end{array}$ & & 0.78 & & 1.02 & & 2.90 \\
\hline standard deviation & & 1.18 & & 1.29 & & 4.25 \\
\hline
\end{tabular}

Table 2: Optimization results 


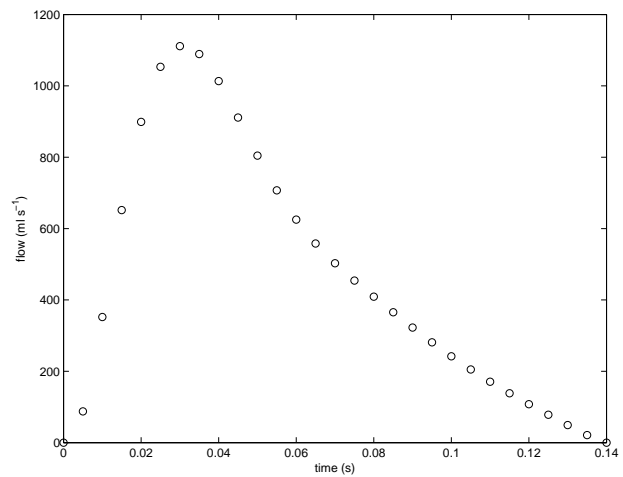

(a) Healthy

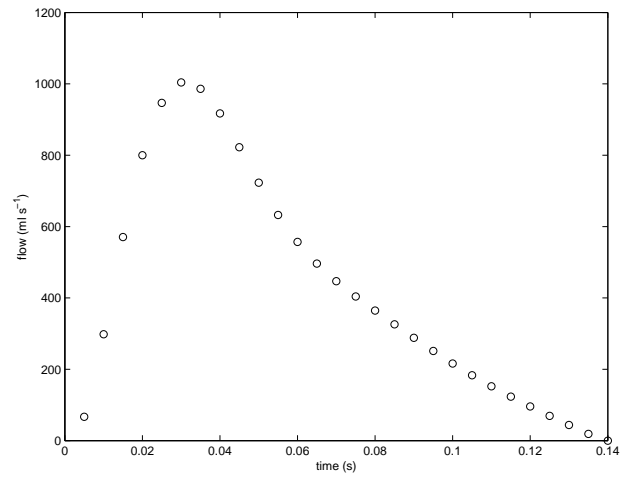

(b) Mitral Stenosis

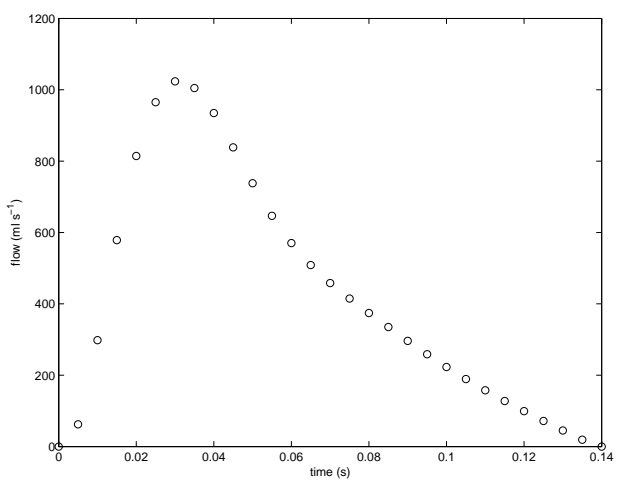

(c) Pulmonary Embolism

Figure 6: Flow through the aortic valve.

\begin{tabular}{|l|l|l|l|}
\hline Noise level (\%) & $\begin{array}{l}\text { Error }(\%) \\
R_{\mathrm{mt}}\end{array}$ & Mean (all parameters) & Standard deviation \\
\hline 5 & 1.8 & 4.3 & 8.2 \\
10 & 2.9 & 5.5 & 7.0 \\
15 & 0.8 & 6.1 & 6.3 \\
\hline
\end{tabular}

Table 3: Percentage error in parameters for Mitral Stenosis with noise 


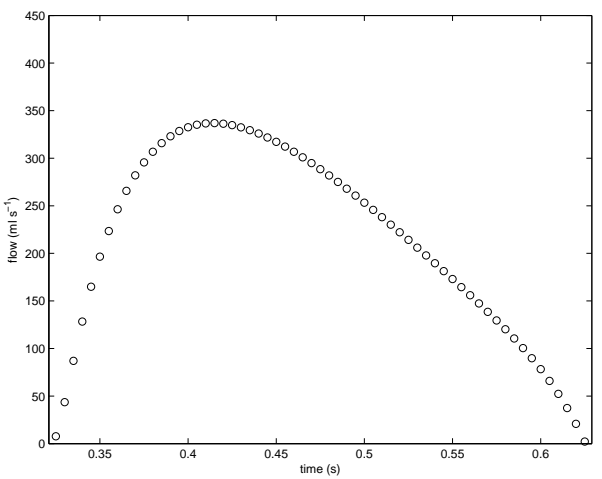

(a) Healthy

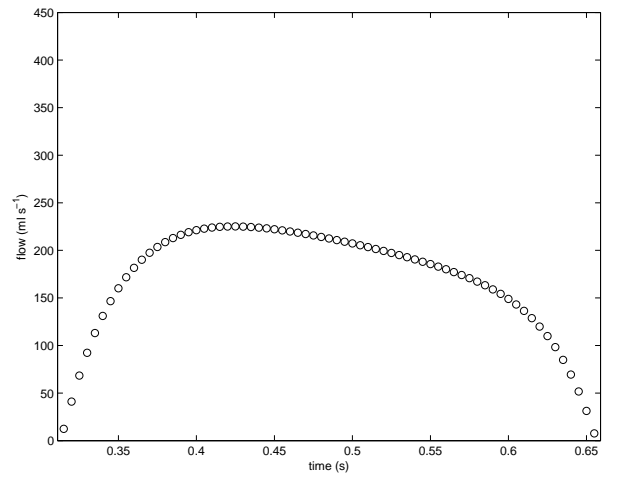

(b) Mitral Stenosis

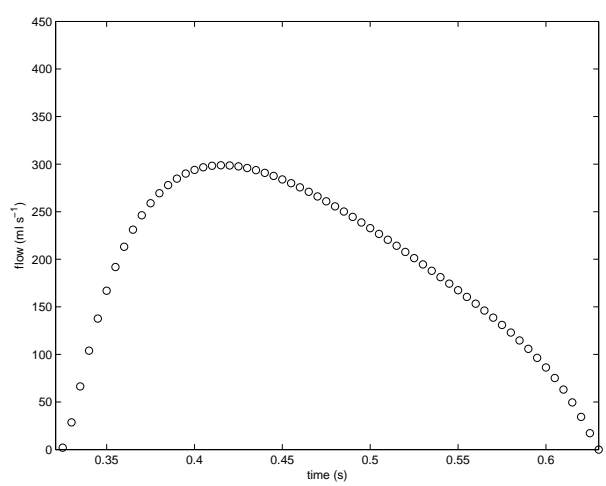

(c) Pulmonary Embolism

Figure 7: Flow through the mitral valve.

\begin{tabular}{|l|l|l|l|}
\hline Noise level (\%) & $\begin{array}{l}\text { Error }(\%) \\
R_{\text {pul }}\end{array}$ & Mean (all parameters) & Standard deviation \\
\hline 5 & 1.5 & 4.4 & 5.6 \\
10 & 3.4 & 4.9 & 5.3 \\
15 & 4.7 & 8.2 & 11.9 \\
\hline
\end{tabular}

Table 4: Percentage error in parameters for Pulmonary Embolism with noise 


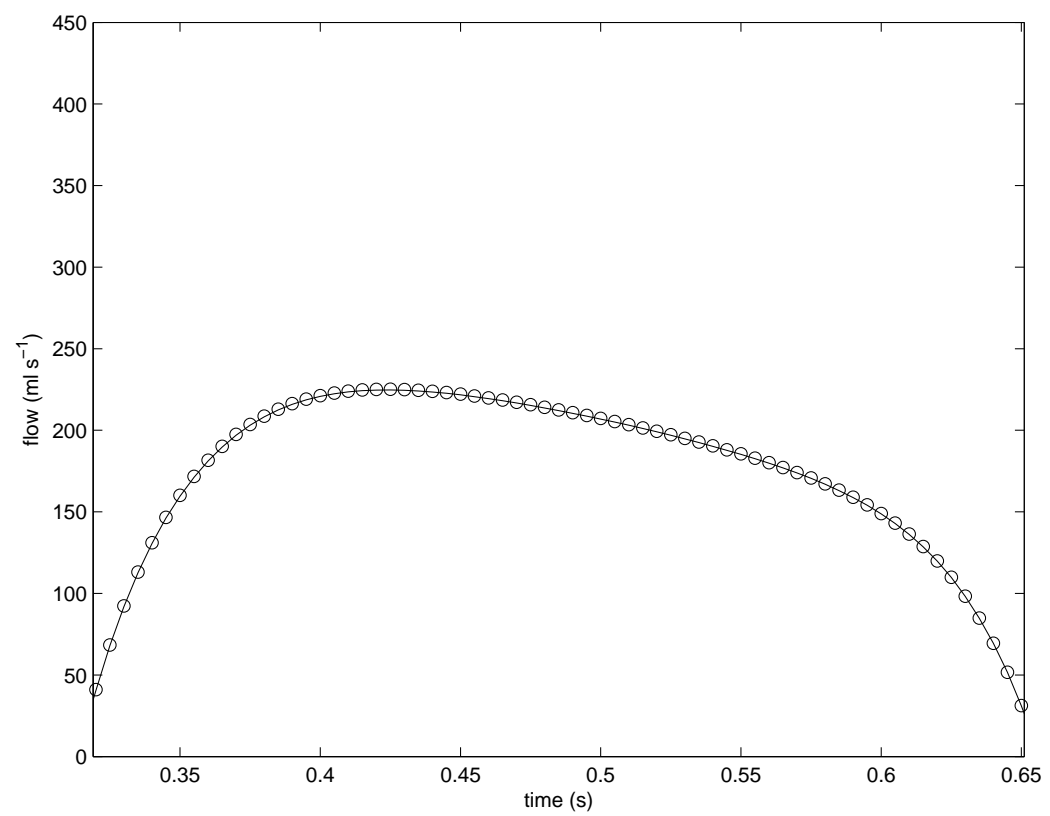

Figure 8: Flow through the mitral valve using identified patient specific parameters (solid line) versus the original true simulation (circles) for Mitral Stenosis 


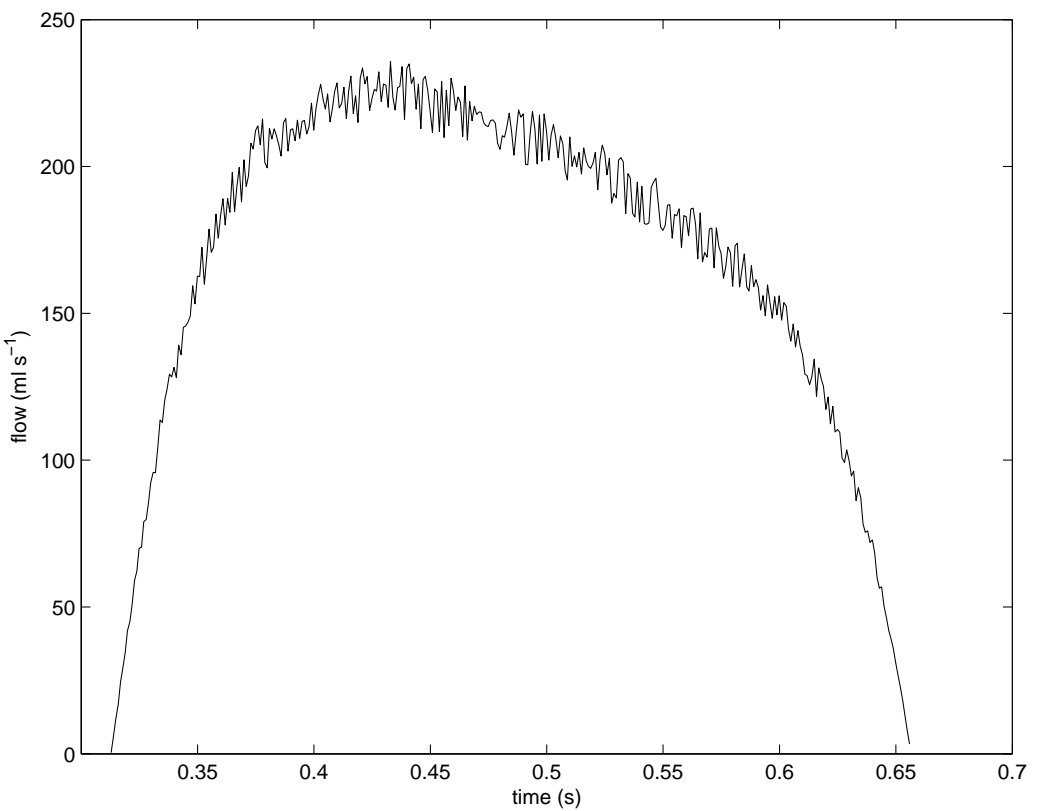

Figure 9: Flow through the mitral valve for Mitral Stenosis with 5\% noise. 